\title{
Ultrasound biomicroscopic study of ciliary body changes in the post-treatment phase of Vogt-Koyanagi-Harada disease
}

\author{
S Wada, T Kohno, N Yanagihara, M Hirabayashi, H Tabuchi, K Shiraki, T Miki
}

Br J Ophthalmol 2002;86:1374-1379

See end of article for authors' affiliations

Correspondence: $\mathrm{Dr}$ Takeya Kohno, Department of Ophthalmology and Visual Science, Osaka City University Graduate School Asahi-machi, 1-4-3, Abeno-ku, Osaka, 545-8585 Japan; takeya@ med.osaka-cu.ac.jp

Accepted for publication 21 May 2002 body in patients with Vogt-Koyanagi-Harada disease.

Methods: Ultrasound biomicroscopy was used to evaluate 14 eyes of seven patients diagnosed with Vogt-Koyanagi-Harada disease. Cross sectional images of the ciliary body and thickness of the pars plana $3.0 \mathrm{~mm}$ posterior to the scleral spur were examined. Predicted thickness of the pars plana was obtained by multiple linear regression analysis of thickness in the acute phase and in the remission phase.

Results: In the active phase, the cross sectional images showed a shallow anterior chamber in eight of the 14 eyes, ciliochoroidal detachment in five eyes, and a thickened ciliary body in all 14 eyes. Internal reflectivity of the ciliary stroma was low, with ciliary processes being unclear in 13 eyes. One month after steroid treatment, slit lamp examination findings were normal in 14 eyes. 10 eyes of five patients were examined by ultrasound biomicroscopy at this stage. Ciliochoroidal detachment was no longer seen in any eye. Internal reflection of the ciliary stroma became relatively homogeneous, and the ciliary processes were seen, though not clearly. However, the pars plana remained thickened. The actual thickness was greater at 1 month after steroid treatment than the predicted thickness for the remission phase. In the remission phase, the internal reflection was homogeneous and the ciliary processes were delineated clearly in all 14 eyes.

Conclusion: Objective, quantitative evaluation of the ciliary body is possible with ultrasound biomicroscopy during the course of Vogt-Koyanagi-Harada disease. Ultrasound biomicroscopy is useful in determining disease activity in the anterior segment and in monitoring the clinical course, and it may improve evaluation of the efficacy of treatment.

V ogt-Koyanagi-Harada disease is a panuveitis with serous retinal detachment and symptoms of meningeal irritation; the disease is accompanied by varying degrees of inflammation of the iris, ciliary body, and choroid. ${ }^{2}$ The associated pathological changes in the ocular fundus have been examined thoroughly by ophthalmoscopy, fluorescein angiography, and ultrasonography. ${ }^{1-4}$ Changes in the anterior portion of the eyeball have been examined in detail by means of slit lamp biomicroscopy. Shallow anterior chambers have been observed in the active phase of the disease, ${ }^{5-7}$ and oedematous swelling has been seen in the ciliary processes by cycloscopy. ${ }^{6}$ However, pathological processes in the ciliary body can be observed only partially by this means, even by those skilled in the observation of scleral depression.

The $50 \mathrm{MHZ}$ ultrasound biomicroscope provides for detailed examination of the anterior segment of the eyeball. The diagnostic utility of ultrasound biomicroscopy has been reported for anterior segment disorders such as glaucoma, iris tumour, corneal diseases, and ocular trauma. ${ }^{89}$ Ciliochoroidal detachment has been detected by ultrasound biomicroscopy in the active stage of Vogt-Koyanagi-Harada disease, ${ }^{10-14}$ and swellings were observed in the pars plicata and pars plana of the ciliary body in two patients, also in the active stage. ${ }^{13}{ }^{14}$ However, ultrasound biomicroscopic features of the ciliary body itself over the course of the disease have not been fully clarified. Thus, we investigated whether changes in the ciliary body in patients with Vogt-Koyanagi-Harada disease could be observed via ultrasound biomicroscopy and used for objective, quantitative evaluation of the disease status.

\section{PATIENTS AND METHODS}

The subjects were seven consecutive patients who were diagnosed with Vogt-Koyanagi-Harada disease at Osaka City University Hospital between November 1996 and December 2000 and observed during the active phase of the disease and for more than 1 year thereafter. The clinical profiles of the seven patients (four men and three women), who ranged in age from 27 to 59 years (mean 44.4 years), are shown in Table 1. The follow up periods ranged from 1 year 10 months to 6 years (mean 39 months).

The diagnosis of Vogt-Koyanagi-Harada disease was dependent upon (1) no history of penetrating ocular trauma or surgery preceding the initial onset of uveitis, (2) binocular iridocyclitis, (3) serous retinal detachment or optic disc hyperaemia or oedema, (4) delayed choroidal filling in the early fluorescein angiography phase and numerous sites of punctate hyperfluorescence caused by dye leakage, and (5) auditory and neurological manifestations (headache, meningismus, and tinnitus) or cerebrospinal fluid pleocytosis. In addition to routine examinations such as slit lamp microscopy and ophthalmoscopy, both eyes of each patient were examined by ultrasound biomicroscopy throughout the active and remission phases of the disease. Remission was defined as the absence of symptoms and abnormal findings observed in the active phase in both the anterior chamber and fundus for 1 year or more after treatment.

Ultrasound biomicroscopy images of the anterior segment are influenced by changes in lighting and also by administration of pilocarpine. ${ }^{15}$ Because it is necessary for the pupil to be consistent in size for follow up examinations, all eyes were examined through dilated pupils with a model 840 ultrasound 
Table 1 Summary of cases

\begin{tabular}{|c|c|c|c|c|c|c|}
\hline Patients & Age & Sex & $\begin{array}{l}\text { Initial visual } \\
\text { acuity }\end{array}$ & $\begin{array}{l}\text { Initial steroid } \\
\text { treatment }\end{array}$ & $\begin{array}{l}\text { Follow up period with } \\
\text { (period of UBM study in } \\
\text { the remission phase) }\end{array}$ & $\begin{array}{l}\text { Final visual } \\
\text { acuity }\end{array}$ \\
\hline 1 & 44 & $\mathrm{~F}$ & $\begin{array}{l}\text { R } 30 \mathrm{~cm} / \mathrm{CF} \\
\mathrm{L} 0.9\end{array}$ & high dose & 73 months (60 months) & $\begin{array}{l}\text { R } 1.2 \\
\text { L } 1.2\end{array}$ \\
\hline 2 & 56 & M & $\begin{array}{l}\text { R } 0.9 \\
\text { L } 0.7\end{array}$ & high dose & 73 months (61 months) & $\begin{array}{l}\text { R } 1.0 \\
\text { L } 1.2\end{array}$ \\
\hline 3 & 59 & M & $\begin{array}{l}\text { R } 1.2 \\
L 1.0\end{array}$ & oral & 32 months (19 months) & $\begin{array}{l}\text { R } 1.5 \\
L 1.0\end{array}$ \\
\hline 4 & 48 & $\mathrm{~F}$ & $\begin{array}{l}\text { R } 1.0 \\
\text { L } 1.2\end{array}$ & pulse & 25 months (12 months) & $\begin{array}{l}\text { R } 1.2 \\
\text { L } 1.2\end{array}$ \\
\hline 5 & 33 & $\mathrm{~F}$ & $\begin{array}{l}\text { R } 1.5 \\
\text { L } 1.0\end{array}$ & oral & 25 months (12 months) & $\begin{array}{l}\text { R } 1.5 \\
\text { L } 1.2\end{array}$ \\
\hline 6 & 45 & M & $\begin{array}{l}\text { R } 0.6 \\
L 0.6\end{array}$ & pulse & 25 months (25 months) & $\begin{array}{l}\text { R } 1.0 \\
\text { L } 1.2\end{array}$ \\
\hline 7 & 27 & M & $\begin{array}{l}\text { R } 0.7 \\
\text { L } 0.3\end{array}$ & pulse & 25 months (25 months) & $\begin{array}{l}\text { R } 1.5 \\
\text { L } 1.0\end{array}$ \\
\hline
\end{tabular}

UBM = ultrasound biomicroscopy.

High dose = betamethazone $20 \mathrm{mg} /$ day as the first dose for 3 days, then tapering; pulse =

methylpredonisolone $1000 \mathrm{mg} /$ day for a period of 3 days, followed by $3 \mathrm{mg}$ of predonisolone, then tapering; oral = predonisolone $30 \mathrm{mg} /$ day as the first dose for $1 \mathrm{month}$, then tapering. biomicroscope and a $50 \mathrm{MHZ}$ transducer (Humphrey Instruments, Inc, San Leandro, CA, USA). Images extending from the iris root to the pars plicata and pars plana were obtained from four directions-that is, at the 12, 3, 6, and 9 o'clock positions. The depth of the anterior chamber, the ciliary body in cross section, and the thickness of the pars plana were examined.

Since results obtained by a single examiner are reportedly more reproducible than those obtained by more than one examiner, ${ }^{16}$ one of us (TK) performed the ultrasound biomicroscopic examinations throughout the study. We removed the water storage cup attached to the biomicroscope apparatus because the cup restricted the visual field of the examiner and made it difficult to keep the conditions of the examinations constant. Without the cup, physiological saline was pooled in an adhesive drape, as is done for ultrasonographic examination by the immersion method. The eyelid was kept open with a lid retractor, and the position of the eyeball was monitored throughout the examination.

The anterior chamber depth was defined at the centre of the chamber as the distance between the posterior surface of the cornea and the anterior surface of the lens on cross sectional images. When the depth of the anterior chamber was more than $0.15 \mathrm{~mm}$ less in the active phase than in the remission phase, ${ }^{12}$ the eye was said to have had a shallow anterior chamber in the active phase. Thicknesses of the pars plana and sclera were measured during the follow up examinations along a line that was drawn perpendicular to the surface of the sclera at a point $3.0 \mathrm{~mm}$ posterior to the scleral spur (Fig lA). That point, $3.0 \mathrm{~mm}$ posterior to the scleral spur, was chosen for
A

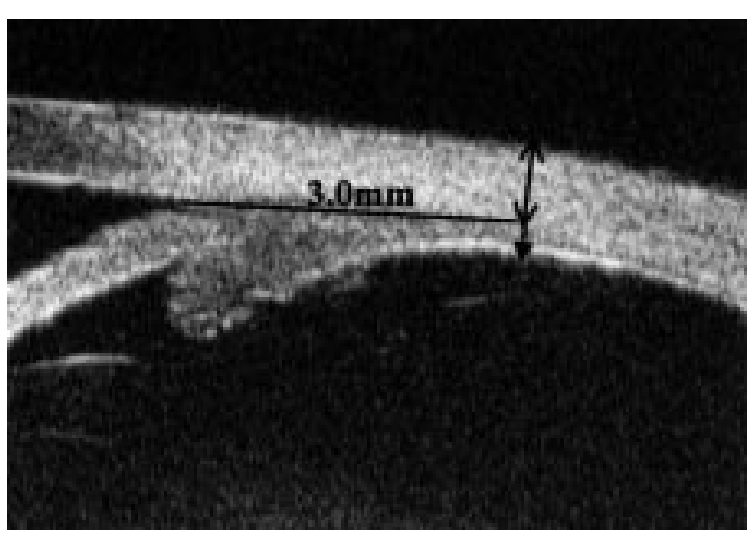

Figure 1 Measurement of the pars plana and sclera thicknesses. (A) Measurements are made from a line dropped from a point $3 \mathrm{~mm}$ posterior to the scleral spur and perpendicular to the scleral surface. The line with the solid arrowhead indicates the thickness of the pars plana, and that marked on both ends indicates the thickness of the sclera. (B) Densitometer curve. The horizontal axis represents the number of pixels from the point of origin located arbitrarily just in front of the eyeball. The vertical axis represents the ultrasound intensity. The mean number of pixels corresponding to points $A$ and $A^{\prime}$ is taken to represent the position of the surface of the sclera on the horizontal axis. Similarly, that corresponding to points $B$ and $B^{\prime}$ is taken to represent the horizontal position of the border between the sclera and the pars plana and that corresponding to points $C$ and $C^{\prime}$ as the horizontal position of the surface of the pars plana. The thickness of the sclera is expressed by the number of pixels between the scleral surface and the sclera-pars plana border (open arrowheads). Similarly, the thickness of the pars plana is expressed by the number of pixels between the sclera-pars plana border and the surface of the pars plana (solid arrowheads). 
Table 2 Ultrasound biomicroscopic findings in the active stage of illness

\begin{tabular}{|c|c|c|c|c|c|c|c|c|}
\hline Patient & Eye & $\begin{array}{l}\text { Dot echo } \\
\text { in anterior } \\
\text { chamber }\end{array}$ & $\begin{array}{l}\text { Dot echo } \\
\text { in vitreous }\end{array}$ & $\begin{array}{l}\text { Shallow } \\
\text { anterior } \\
\text { chamber* }\end{array}$ & $\begin{array}{l}\text { Ciliochoroidal } \\
\text { detachment }\end{array}$ & $\begin{array}{l}\text { Low reflectivity } \\
\text { of internal } \\
\text { echo }\end{array}$ & $\begin{array}{l}\text { Unclear image } \\
\text { of ciliary } \\
\text { processes }\end{array}$ & $\begin{array}{l}\text { Increased } \\
\text { thickness of } \\
\text { pars plana }\end{array}$ \\
\hline \multirow[t]{2}{*}{1} & $R$ & - & + & + & $2+$ & + & + & + \\
\hline & L & - & + & + & $2+$ & + & + & + \\
\hline \multirow[t]{2}{*}{2} & $R$ & $2+$ & $2+$ & - & - & + & + & + \\
\hline & L & + & + & + & - & + & + & + \\
\hline \multirow[t]{2}{*}{3} & $R$ & $2+$ & $2+$ & + & - & + & + & + \\
\hline & L & + & + & + & - & + & + & + \\
\hline \multirow[t]{2}{*}{4} & $R$ & + & + & - & - & + & + & + \\
\hline & L & + & + & - & - & + & + & + \\
\hline \multirow[t]{2}{*}{5} & $R$ & $2+$ & + & + & + & + & + & + \\
\hline & $\mathrm{L}$ & $2+$ & + & + & + & + & + & + \\
\hline \multirow[t]{2}{*}{6} & $R$ & $2+$ & + & - & - & + & + & + \\
\hline & L & $2+$ & + & - & + & + & + & + \\
\hline \multirow[t]{2}{*}{7} & $R$ & + & + & + & - & + & + & + \\
\hline & $\mathrm{L}$ & + & + & - & - & - & - & + \\
\hline
\end{tabular}

accurate comparison of thickness measurements because points $1.0-2.0 \mathrm{~mm}$ posterior to the scleral spur were in the pars plicata portion; thus their images were subject to change during examination. Points more than $4.0 \mathrm{~mm}$ posterior to the scleral spur were situated at the peripheral area in a $5 \times 5 \mathrm{~mm}$ field of the ultrasound biomicroscopy images. With image analysis software (NIH Image, version 1.58), the brightness along the line described above was expressed in 256 gradations and plotted as shown in Figure 1B. A border between the sclera and the pars plana and a border between the pars plana and the vitreous were determined as shown in Figure 1B. Thus, the thickness of the sclera was defined as the distance between the external surface of the sclera and the sclera-pars plana border, and the thickness was expressed as the number of pixels between these two borders. The thickness of the pars plana was defined similarly as the distance between the sclera-pars plana border and the pars plana-vitreous border and was expressed as the number of pixels.

Because cross sectional ultrasound biomicroscopy images of the ciliary body can vary according to the angle of the probe directed toward the eyeball, reproducibility of our ultrasound biomicroscopic examinations was assessed in the following manner. The examiner repeated the ultrasound biomicroscopic measurements five times in each of five eyes of five healthy volunteers, and another examiner (SW) calculated the coefficients of variation obtained for the thicknesses of the pars plana and the sclera. The within group variation obtained for the thickness of the pars plana in normal eyes was 3.68\% and the between group variation was $26.0 \%$. Within group variation for the sclera in the normal eyes was $1.63 \%$ and between group variation was $12.9 \%$. Thus, good reproducibility was obtained.

The paired $t$ test was used for statistical comparison of the pars plana thicknesses, which were obtained in all 14 eyes of the seven study patients, between the active and remission phases. In addition, in 10 eyes of five patients in whom ultrasound biomicroscopy examination was performed 1 month after the start of steroid treatment, one way analysis of variance with a least significant difference test was used for statistical comparison of the thicknesses obtained in the active phase, 1 month after steroid treatment and in the remission phase. Statistical computations were done on a personal computer with spss $9.0 \mathrm{~J}$ (SPSS Inc, Chicago, IL, USA). A p value of less than 0.01 was considered significant.
Because the thickness of pars plana can vary even among healthy individuals, a general treatment goal can not be set for the pars plana thickness. Thus, for each patient, we predicted the pars plana thickness during remission for objective evaluation of the changes in the pars plana thickness over the course of the disease. The prediction was done by multiple linear regression analysis of thickness in the active phase and in the remission phase in 14 eyes of the seven study patients. We calculated the predicted value and 95\% confidence interval in the remission phase using the regression line, and the predicted value was compared with the actual thickness of the pars plana measured at 1 month after steroid therapy.

\section{RESULTS}

The results of ultrasound biomicroscopy during the acute stage of illness are summarised in Table 2. Shallow anterior chambers were observed in eight of the 14 eyes during the active phase, and ciliochoroidal detachment was observed in five eyes during this phase. The anterior chamber was shallow in four of the five eyes with ciliochoroidal detachment. Cross sectional images showed a thickened ciliary body in all 14 eyes. In 13 eyes, internal echo reflections of the ciliary body were weak, and the ciliary processes were not delineated clearly. One month after steroid treatment, the depth of the anterior chamber appeared normal in all 14 eyes under slit lamp examination, and no inflammatory cells were found in the anterior chamber of any eye. At this stage, 10 eyes of five patients were examined by ultrasound biomicroscopy. Ciliochoroidal detachment, which was observed in the active phase in four of these 10 eyes, was no longer detectable in any eye. The depth of the anterior chamber was normal, and dot echoes were not noted in the anterior chamber in any of the 10 eyes. Internal echoes of the ciliary stroma became relatively homogeneous, and the ciliary processes were seen, though not clearly. On cross sectional images, the ciliary body remained thickened. During the remission phase, ultrasound biomicroscopic examination revealed no ciliochoroidal detachment in any of the 14 eyes. On cross sectional images, the ciliary body appeared thinner. The internal echo reflection was more homogeneous, and the ciliary processes were delineated clearly.

The pars plana was significantly thick in the 14 eyes during the active phase (19.15 (SD 5.26) pixels) in comparison with the remission phase $(10.56(2.48)$ pixels $)(p<0.0001)$. No 
Table 3 Thickness of the pars plana on follow up examination and its predicted thickness in the remission phase

\begin{tabular}{|c|c|c|c|c|c|c|c|c|}
\hline \multirow[b]{2}{*}{ Patient } & \multirow[b]{2}{*}{ Eye } & \multicolumn{5}{|c|}{ Measured thickness of the pars plana (unit: pixel) } & \multicolumn{2}{|c|}{$\begin{array}{l}\text { Predicted thickness of the } \\
\text { pars plana in the remissior } \\
\text { phase (unit: pixel) }\end{array}$} \\
\hline & & $\begin{array}{l}\text { Acute } \\
\text { phase }\end{array}$ & & 1 month & & $\begin{array}{l}\text { Remission } \\
\text { phase }\end{array}$ & $\begin{array}{l}\text { Predicted } \\
\text { value }\end{array}$ & $95 \% \mathrm{Cl}$ \\
\hline \multirow[t]{2}{*}{1} & $\mathrm{R}$ & 28.6 & $\rightarrow$ & * & $\rightarrow$ & 11.7 & 13.8 & 9.4 to 18.1 \\
\hline & L & 26.8 & $\rightarrow$ & * & $\rightarrow$ & 13.4 & 13.2 & 9.0 to 17.4 \\
\hline \multirow[t]{2}{*}{2} & $\mathrm{R}$ & 17.3 & $\rightarrow$ & * & $\rightarrow$ & 10.2 & 10.4 & 6.5 to 14.4 \\
\hline & L & 23.8 & $\rightarrow$ & * & $\rightarrow$ & 12.7 & 12.3 & 8.3 to 16.4 \\
\hline \multirow[t]{2}{*}{3} & $\mathrm{R}$ & 15.5 & $\rightarrow$ & 10.1 & $\rightarrow$ & 8.5 & 9.9 & 5.9 to 13.9 \\
\hline & L & 9.5 & $\rightarrow$ & 9.4 & $\rightarrow$ & 8.4 & 8.1 & 3.8 to 12.6 \\
\hline \multirow[t]{2}{*}{4} & $\mathrm{R}$ & 20.9 & $\rightarrow$ & 16.4 & $\rightarrow$ & 10.4 & 11.5 & 7.5 to 15.4 \\
\hline & L & 10.9 & $\rightarrow$ & 9.9 & $\rightarrow$ & 5.5 & 8.5 & 4.3 to 12.8 \\
\hline \multirow[t]{2}{*}{5} & $\mathrm{R}$ & 19.8 & $\rightarrow$ & 16.7 & $\rightarrow$ & 12.0 & 11.2 & 7.2 to 15.1 \\
\hline & L & 21.2 & $\rightarrow$ & 14.1 & $\rightarrow$ & 10.1 & 11.6 & 7.6 to 15.5 \\
\hline \multirow[t]{2}{*}{6} & $\mathrm{R}$ & 18.1 & $\rightarrow$ & 14.5 & $\rightarrow$ & 13.1 & 10.7 & 6.7 to 14.6 \\
\hline & L & 18.9 & $\rightarrow$ & 14.0 & $\rightarrow$ & 11.9 & 10.9 & 7.0 to 14.8 \\
\hline \multirow[t]{2}{*}{7} & $\mathrm{R}$ & 19.4 & $\rightarrow$ & 13.7 & $\rightarrow$ & 12.5 & 11.0 & 7.1 to 15.0 \\
\hline & $\mathrm{L}$ & 17.4 & $\rightarrow$ & 15.3 & $\rightarrow$ & 13.2 & 10.5 & 6.5 to 14.4 \\
\hline
\end{tabular}

significant difference was found in the thickness of the sclera between the active (41.5 (3.35) pixels) and remission phase (40.03 (3.16) pixels) $(\mathrm{p}=0.0814)$. In the 10 eyes that were examined by ultrasound biomicroscopy at 1 month after steroid treatment, the thickness of the pars plana in the active phase differed significantly from that observed at 1 month after steroid treatment $(p=0.0002)$. Further, there was a statistical difference between the thickness of the pars plana measured at 1 month after steroid treatment and that during the remission phase $(\mathrm{p}=0.0016)$.

The correlation coefficient of the regression line was 0.679273 , and the equation y (predicted value in the remission phase $)=5.33335+0.294416 \times($ actual thickness in the active phase) describes the regression line $(p=0.0075)$. The predicted value in the remission phase ranged from 8.1 to 13.8 (Table 3 ). In all 10 eyes that were examined by ultrasound biomicroscopy at 1 month after steroid treatment, the actual thickness was greater at 1 month after steroid treatment than the predicted thickness in the remission phase.

\section{TWO REPRESENTATIVE CASES \\ Case 1}

The patient was a 44 year old woman with blurred vision. She had experienced headache, nausea, and back stiffness for 4 days. Corrected visual acuity was counting fingers at $30 \mathrm{~cm}$ in the right eye and 0.9 in the left eye. The number of lymphocytes in the cerebrospinal fluid was increased. The anterior chambers of both eyes were shallow and without inflammatory cells. Serous retinal detachment was found bilaterally at the posterior pole. Fluorescein angiography showed delayed choroidal filling in the early phase and numerous sites of punctate fluorescein hyperfluorescence as well as diffusion of the fluorescein dye into the subretinal space (Fig 2A). On ultrasound biomicroscopic examination, shallow anterior chambers were noted in both eyes without dot echoes within. Cross sectional images of the ciliary body showed thickening and marked ciliochoroidal detachment (Fig 2B). The patient was started on high dose corticosteroid treatment, betamethasone sodium phosphate at $20 \mathrm{mg} /$ day. The dosage was decreased gradually over a period of 2 years and then gradually withdrawn. During the course of treatment, there was no inflammation in the anterior segment or fundus, and the fundus gave a sunset glow appearance at the end of the treatment. The patient had no recurrence in either the anterior or posterior segments during the 5 years after high dose corticosteroid treatment and was thus thought to be in remission. The cross sectional images of the ciliary body were thinner than they were in the active phase, and the internal echo was homogeneous. The ciliary processes were delineated clearly (Fig 2C). The pars plana of the right eye decreased in thickness from 28.6 pixels in the active phase to 11.7 pixels in the remission phase, and that of the left eye decreased similarly from 26.8 pixels to 13.4 pixels.

\section{Case 4}

The patient was a 48 year old woman with headache, tinnitus, and blurred vision. Corrected visual acuity was 1.0 in the right eye and 1.2 in the left. Intraocular pressure was $18 \mathrm{~mm} \mathrm{Hg}$ in the right eye and $16 \mathrm{~mm} \mathrm{Hg}$ in the left. The anterior chamber was shallow, and inflammatory cells were observed in both eyes. Serous retinal detachment was found bilaterally at the posterior pole. Fluorescein angiography showed delayed choroidal filling, numerous sites of punctate hyperfluorescence, and diffusion of the fluorescent dye into the subretinal space. Ciliochoroidal detachment was not noted on ultrasound biomicroscopic examination. Cross sectional images showed a markedly thickened ciliary body and low echo regions in the ciliary stroma of both eyes. The ciliary processes were not delineated. The patient underwent pulse steroid treatment in which methylprednisolone sodium phosphate was administered intravenously at $1000 \mathrm{mg} /$ day for 3 days. Thereafter, oral prednisolone sodium phosphate was given at $30 \mathrm{mg} /$ day for 1 month and then gradually reduced. After 6 months, when the prednisolone sodium phosphate was being given at $5 \mathrm{mg} / \mathrm{day}$, inflammation recurred in the anterior segment of both eyes. The anterior chambers were slightly shallow, and inflammatory cells were seen within the anterior chamber and vitreous. The optic discs of both eyes were reddish and slightly oedematous. Fluorescein angiography showed bilateral leakage of the dye at the optic disc. On ultrasound biomicroscopic examination, the anterior chambers of both eyes were slightly shallow and showed dot echoes. The cross sectional images 

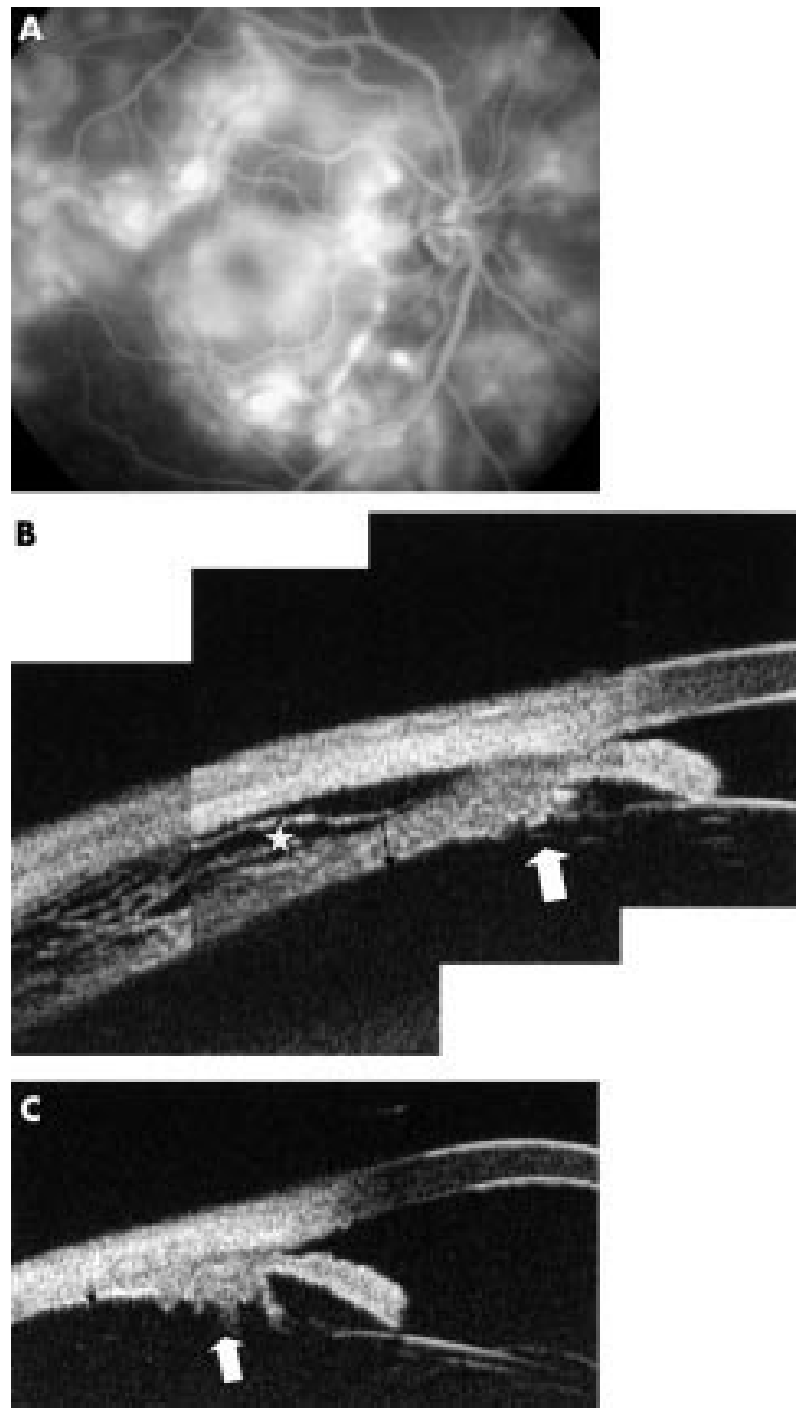

Figure 2 Case 1. (A) A late phase (3 minute) fluorescein angiogram. Numerous sites of punctate hyperfluorescence caused by fluorescein dye leakage from the retinal pigment epithelium and diffusion of the dye beneath the retina are observed. (B) Ultrasound biomicroscopic image obtained when the patient was first examined. Ciliochoroidal detachment is evident (asterisk). The pars plicata and pars plana are thickened, and the ciliary processes are not clear (thick arrow). Thin arrow: Pars plana thickness is 28.6 pixels. (C) Ultrasound biomicroscopic image obtained 5 years after initial examination. Ciliochoroidal detachment is not detected. The ciliary processes are delineated clearly (thick arrow). Thin arrow: Pars plana thickness is 11.7 pixels.

showed the ciliary body to be markedly thickened, and low echo regions were observed. The ciliary processes were not delineated (Fig 3A). Ten days after the prednisolone sodium phosphate was increased to $30 \mathrm{mg} /$ day, the anterior chamber was of normal depth, and the inflammatory cells in the anterior chamber and vitreous had disappeared. On ultrasound biomicroscopic examination performed 1 month after the prednisolone sodium phosphate was increased, the ciliary processes were apparent, but the ciliary body remained thickened on the cross sectional images (Fig 3B). One year after the relapse, the cross sectional images of the ciliary body appeared thinner, and the internal echo showed homogeneous reflection. The ciliary processes of the ciliary body were distinct (Fig 3C). Thickness of the pars plana in the active phase, 1 month after steroid treatment, and in the remission phase was 20.9 pixels, 16.4 pixels, and 10.4 pixels, respectively, in the right eye, and 10.9 pixels, 9.9 pixels, and 5.5 pixels, respectively, in
A
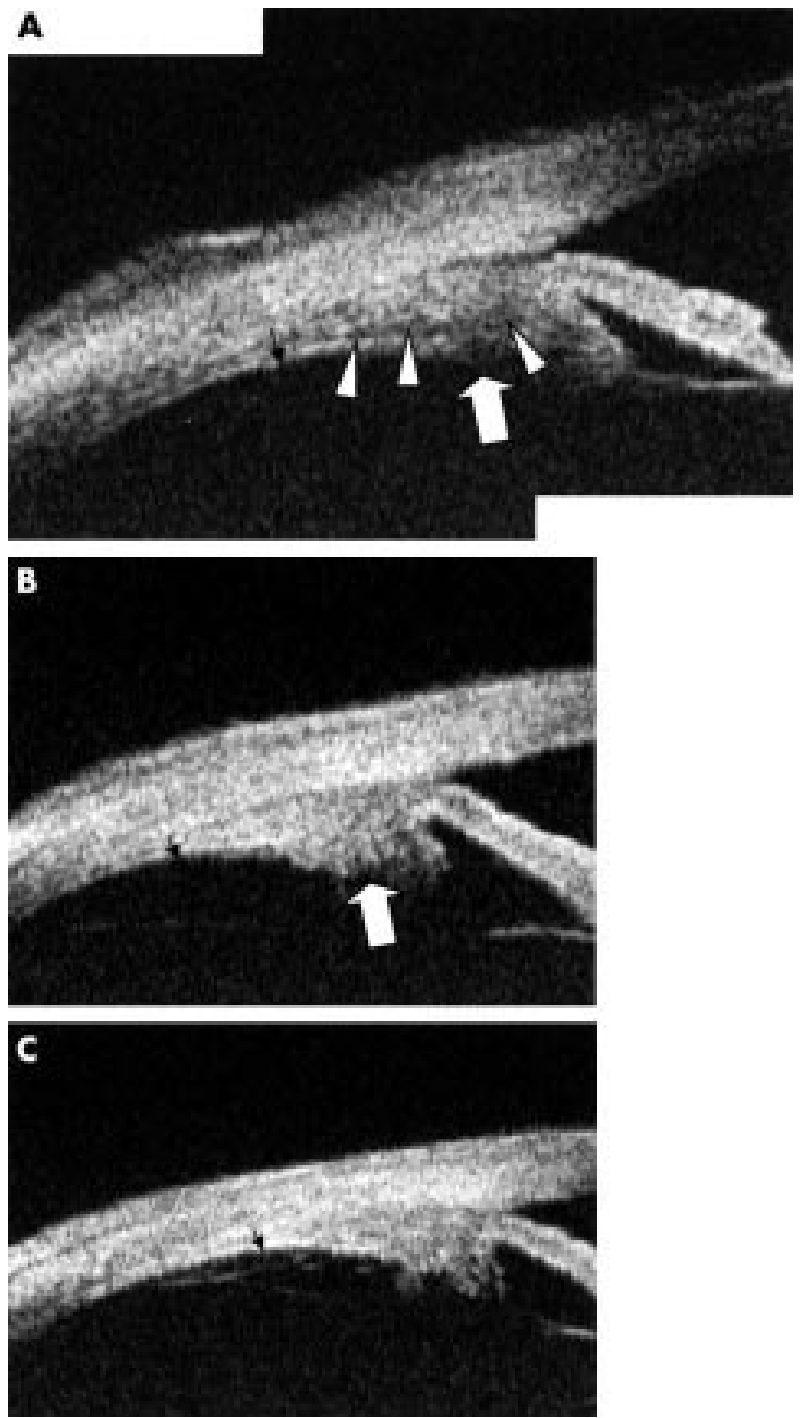

Figure 3 Case 4. (A) Ultrasound biomicroscopic image obtained upon relapse. Ciliochoroidal detachment is not observed. The pars plicata and pars plana are thickened (thick arrow). Low echo regions are seen in several places in the ciliary stroma (arrowheads). Thin arrow: Pars plana thickness is 10.9 pixels. (B) Ultrasound biomicroscopic image obtained 1 month after steroid treatment. The pars plicata and pars plana are slightly thickened, and the ciliary processes are delineated, although only vaguely. The internal echo is homogeneous (thick arrow). Thin arrow: pars plana thickness is 9.9 pixels. (C) Ultrasound biomicroscopic image obtained 1 year after the start of steroid treatment. The ciliary processes are observed clearly, and the internal echo is homogeneous. Thin arrow: pars plana thickness is 5.5 pixels.

the left eye. The predicted value for the remission phase was 11.5 pixels in the right eye and 8.5 pixels in the left eye.

\section{DISCUSSION}

In Vogt-Koyanagi-Harada disease, histopathological infiltration of inflammatory cells into the iris, ciliary body, and choroid is found. ${ }^{18} 19$ The inflammatory changes presumably cause the ciliary body thickening, the decreased internal echo reflection of the ciliary body stroma, the obscured appearance of the ciliary processes, and the ciliochoroidal detachment observed on ultrasound biomicroscopic examination, all of which were seen in the present series. At 1 month after the start of steroid treatment, slit lamp examinations showed that the anterior chamber returned to normal depth and became cell-free in all 14 eyes. In both eyes of the five patients who 
were examined under ultrasound biomicroscopy at 1 month after the start of steroid treatment, the ciliary body remained thickened; ciliary processes were only vaguely delineated; and the internal echo of the ciliary stroma became relatively, but not completely, homogeneous. Thus, the ultrasound biomicroscopy abnormalities seen in the acute stage of illness remained to various degrees 1 month after the start of steroid treatment. Even during the stage in which slit lamp examination indicated amelioration of inflammation in the anterior segment, ultrasound biomicroscopy detected some remaining inflammation in the ciliary body.

Because objective, quantitative analysis of the inflammatory abnormalities remaining during steroid treatment is difficult, the thickness of the pars plana and that of the sclera were measured, both $3.0 \mathrm{~mm}$ posterior to the scleral spur. As stated above, the pars plana was significantly thicker in the active phase than in the remission phase, and no difference was found in the scleral thickness, indicating that thickening of the ciliary body in the active phase and its regression in the remission phase were not due to fluctuations in examination conditions. Rather, they represented development and recovery of actual changes in the ciliary body in the presence of Vogt-Koyanagi-Harada disease.

Furthermore, the pars plana was shown to decrease gradually in thickness from the active phase to 1 month after steroid treatment and then further in the remission phase; differences between succeeding periods were statistically significant. To evaluate the presence or absence of remaining changes in the ciliary body in the follow up period objectively, we obtained the regression line of the pars plana thickness in the 14 eyes of the present seven patients and calculated the predicted remission phase value for each eye. Since variation in the $95 \%$ confidence interval was too broad, probably due to the small number of patients, we compared the actual thickness at 1 month after steroid treatment and the predicted remission phase value. In all 10 eyes, the actual thickness measured was greater at 1 month after steroid treatment than the predicted remission phase thickness. Thus, predicting the thickness at the pars plana portion is helpful for estimating remaining inflammatory changes in the ciliary body, and further studies are needed to clarify whether this measure can be an indicator for determining when and how to taper the steroid. The regression line and predictive values obtained in this study were not of certain reliability for the following reasons. The first is the wide 95\% confidence interval we discussed above. Secondly, the pars plana thickness may vary greatly in the acute phase, depending on the severity of the inflammation, and the time to remission could vary on a case by case basis. Thirdly, since indocyanine green angiography showed choroidal abnormalities even when disease activity appeared ameliorated via ophthalmoscopy and fluorescein angiography studies, ${ }^{20}$ the ciliary body might be thickened even in the remission phase. Thus, further studies involving a greater number of patients and comparisons between pars plana thickness in the remission phase and that of normal subjects are needed to improve the accuracy of predictive values obtained by regression analysis.

In summary, we showed changes in the ciliary body itself manifested by thickening of the pars plicata and pars plana, low echo lesions in the ciliary stroma, vague images of the ciliary processes, and quantitatively increased thickness of the pars plana using ultrasound biomicroscopy. These changes were alleviated as disease activity decreased but continued in varying degrees after 1 month, even when slit lamp microscopic examination indicated resolution of the disease. Because recurrence of iridocyclitis during the course of the disease can bring about complications such as secondary glaucoma, cataract, and fundus diseases such as choroidal neovascularisation and chorioretinal atrophy, ${ }^{1-3}{ }^{21}$ objective evaluation of disease activity in the anterior segment of the eye in cases of Vogt-Koyanagi-Harada disease is important, as is that in the posterior portion via fluorescein and indocyanine green angiography. We conclude that ultrasound biomicroscopy is useful for determining disease activity and for monitoring the clinical course of Vogt-Koyanagi-Harada disease, and we hope that ultrasound biomicroscopy may play a part in improvement of evaluation of the efficacy of treatment.

\section{ACKNOWLEDGEMENT}

This study was supported in part by the Fund for Medical Research from Osaka City University Medical Research Foundation, Osaka, Japan.

\section{Authors' affiliations}

S Wada, T Kohno, M Hirabayashi, H Tabuchi, K Shiraki, T Miki, Department of Ophthalmology and Visual Science, Osaka City University Graduate School of Medicine, Osaka, Japan

N Yanagihara, Department of Ophthalmology, Jyuso Municipal Hospital, Japan

\section{REFERENCES}

1 Sugiura S. Vogt-Koyanagi-Harada disease. Jpn J Ophthalmol 1978;22:9-35

2 Moorthy RS, Inomata H, Rao NA. Vogt-Koyanagi-Harada syndrome. Surv Ophthalmol 1995;39:265-92.

3 Rubsamen PE, Gass JDM. Vogt-Koyanagi-Harada syndrome:clinical course, therapy, and long-term visual outcome. Arch Ophthalmol 1991; 109:682-7.

4 Forster DJ, Cano MR, Green RL, et al. Echographic features of the Vogt-Koyanagi-Harada syndrome. Arch Ophthalmol 1990;108:1421-6.

5 Kimura R, Sakai M, Okabe H. Transient shallow anterior chamber as initial symptom in Harada's syndrome. Arch Ophthalmol 1981;99:1604-6.

6 Kimura R, Kasai M, Shoji K, et al. Swollen ciliary processes as an initial symptom in Vogt-Koyanagi-Harada syndrome. Am J Ophthalmol 1983;95:402-3

7 Tokuoka S, Iwasaki Y, Sugasawa J. Harada's disease with shallow anterior chamber, myopia and intraocular pressure rise in a pair of siblings. Jpn Clin Ophthalmol 1988;42:365-8.

8 Pavlin CJ, Harasiewicz K, Sherar MD, et al. Clinical use of ultrasound biomicroscopy. Ophthalmology 1991;98:287-95.

9 Pavlin CJ, Foster FS. Part 2. Ultrasound biomicroscopy of ocular disease. In:Ultrasound biomicroscopy of the eye. New York: Springer-Verlag 1995:63-208

10 Ashizuka S, Nishioka Y, Kawano Y, et al. Ciliochoroidal detachment in Vogt-Koyanagi-Harada syndrome. Jpn J Clin Ophthalmol 1997;51:1067-70.

11 Kawano Y, Tawara A, Nishioka Y et al. Ultrasound biomicroscopic analysis of transient anterior chamber in Vogt-Koyanagi-Harada syndrome. Am J Ophthalmol 1996;121:720-3.

12 Maruyama Y, Kimura Y, Kishi S, et al. Serous detachment of the ciliary body in Harada disease. Am J Ophthalmol 1998;125:666-72.

13 Kishi A, Naoi N, Sawada A. Ultrasound biomicroscopic findings of acute angle-closure glaucoma in Vogt-Koyanagi-Harada syndrome. Am J Ophthalmol 1996; 122:735-7.

14 Gentile RC, Berinstein DM, Liebmann J, et al. High-resolution ultrasound biomicroscopy of the pars plana and peripheral retina. Ophthalmology 1998; 105:478-84.

15 Pavlin CJ, Foster FS. Plateau iris syndrome:changes in angle opening associated with dark, light, and pilocarpine administration. Am J Ophthalmol 1999;128:288-91.

16 Tello CJ, Liebmann J, Potash SD, et al. Measurement of ultrasound biomicroscopy images:intraobserver and interobserver reliability. Invest Ophthalmol Vis Sci 1994;35:3549-52.

17 Ueda J, Shirakashi M, Sawaguchi S, et al. Reproducibility of ultrasound biomicroscopy:measurement of anterior segment structures in normal eyes. J Eye (Atarashii Ganka) 1996;13:609-12.

18 Ikui H, Hiroshi M, Furuyoshi Y. Two cases of idiopathic uveitis. Acta Soc Ophthalmol Jpn 1952;56:1079-91.

19 Ikvi H, Hiyama H. Clinical and experimental studies on idiopathic uveitis. Acta Soc Ophthalmol Jpn 1956;60:1687-95.

20 Kohno T, Miki T, Shiraki K, et al. Subtraction ICG angiography in Harada's disease. Br J Ophthalmol 1999:83:822-33.

21 Sonoda S, Nakao K, Ohba N. Extensive chorioretinal atrophy in Vogt-Koyanagi-Harada disease. Jpn J Ophthalmol 1999;43: $113-19$. 